\title{
Biomechanical study of the funnel technique applied in thoracic pedicle screw replacement
}

\author{
Yi-Jiang Huang, Mao-Xiu Peng, Shao-Qi He, Liang-Le Liu, Ming-Hai Dai, Chenxuan Tang*
}

Department of Orthopaedics, the Third Affiliated Hospital of Wenzhou Medical College, Wenzhou 325200, Zhejiang, China

\begin{abstract}
Background: Funnel technique is a method used for the insertion of screw into thoracic pedicle.

Aim: To evaluate the biomechanical characteristics of thoracic pedicle screw placement using the Funnel technique, trying to provide biomechanical basis for clinical application of this technology.

Methods: 14 functional spinal units (T6 to T10) were selected from thoracic spine specimens of 14 fresh adult cadavers, and randomly divided into two groups, including Funnel technique group $(\mathrm{n}=7)$ and Magerl technique group ( $\mathrm{n}=7$ ). The displacement-stiffness and pull-out strength in all kinds of position were tested and compared.

Results: Two fixed groups were significantly higher than that of the intact state $(\mathrm{P}<0.05)$ in the spinal central axial direction, compression, anterior flexion, posterior bending, lateral bending, axial torsion, but there were no significant differences between two fixed groups $(\mathrm{P}>0.05)$. The mean pull-out strength in Funnel technique group (789.09 \pm 27.33$)$ was lower than that in Magerl technique group $(\mathrm{P}<0.05)$.

Conclusions: The Funnel technique for the insertion point of posterior bone is a safe and accurate technique for pedicle screw placement. It exhibited no effects on the stiffness of spinal column, but decreased the pull-out strength of pedicle screw. Therefore, the funnel technique in the thoracic spine affords an alternative for the standard screw placement.
\end{abstract}

Keywords: Thoracic; Pedicle screws; Biomechanics; Funnel technique

DOI: http://dx.doi.org/10.4314/ahs.v14i3.30

\section{Introduction}

Pedicle screw system has been widely used to support spine, maintain stability, and achieve solid bony fusion in patients with degeneration, fracture and scoliosis. It has superior biomechanical and clinical data when compared with other methods of instrumentation ${ }^{1}$. However, the complications (e.g., less rigidity, loosening of the screw, etc.) were common after operation, especially in patients with osteoporosis ${ }^{2}$. The factors affecting screw stability have been reported to be screw size, insertion depth, insertion technique and also bone mineral density ${ }^{3,4}$.

Magerl technique is defined as the atlantoaxial transarticular screw insertion. Funnel technique is a method used for the screw insertion into thoracic pedicle, in which abrasion drill or bone nibbling forceps

\section{* Corresponding author:}

Chenxuan Tang

Department of Orthopaedics, the Third Affiliated Hospital of

Wenzhou Medical College, Wenzhou 325200, Zhejiang, China

E-mail: tangchenxuanwenz@163.com is used to increase the accuracy of screw insertion ${ }^{5}$. This technology was firstly employed to make the specimen for thoracic vertebrae of cadavers in 2002, and the failure rate was reported to be only $6 \%{ }^{1}$.

To date, no biomechanical study has been given for this technology, resulting in the existence of many contradictions and speculations in this field. Some researchers have speculated that fixation strength might be significantly affected because part of coriaceous and cancellous bone was removed. Additionally, the breach sites of coriaceous are arbitary. All these factors strongly limit the application of funnel technique.

From December of 2008 to August of 2009, the comparison between funnel technique and traditional Magerl technique was performed in 14 cadavers. The fixation strength was compared to evaluate the biomechanical properties of funnel technique.

\section{Materials and Methods}

\section{The preparation of specimen}

This is a cadaveric study involving 14 fresh human cadavers (T1-T12), including 8 male and 6 female. The ages range from 21 to 49, with the average value of 38.9. $\mathrm{X}$-ray was used to exclude the diseases, including spinal tuberculosis, tumors, osteoporosis, and degeneration. The specimen was divided from the intervertebral disk 
of T5/6 and T10/11 to make 14 function spinal units (FSU) every 5 body of vertebra. The paravertebral muscle was completely taken, and ligamental intervertebralia was maintained. The specimen can be stored in $-20^{\circ} \mathrm{C}$ refrigerator for 15-30 day. One day before experiment, the specimen was thawn and the experiment must be finished within $8 \mathrm{~h}$. Each body of vertebra located in the two sides of thoracic vertebra was embedded with polymethylmethacrylate (PMMA), and the central body of vertebra was made for injury model of anterior and central column using Panjabi method. The residual two body of vertebra was used for fixation.

\section{Biomechanical experiment}

Universal material testing machine $(2500 \mathrm{~N}$, Zwick, Germany) was used in this study for loading exposure, and the displacement of the middle vertebral segment was measured using KG-digital grating high precision micrometer. Pre-loading experiment was first carried out to determine the axial center compression points of each functional segment. Loading of $250 \mathrm{~N}$ was applied step by step on the center point and $20 \mathrm{~mm}$ location all around to induce thoracic non-destructive flexion and extension in axis and all four horizontal directions. Pre-loading was performed twice before the data were collected to eliminate creep and relaxation of soft tissues in every assay (Fig. 1).

Fig. 1 The representative figures for Biomechanical experiment. Universal material testing machine $(2500 \mathrm{~N}$, Zwick, Germany) was used in this study for loading exposure and the displacement of the middle vertebral segment was measured using KG-digital grating high precision micrometer. (A) displacement-stiffness test; (B) torsion test; (C)pullout strength test

\section{Fig. 1A}

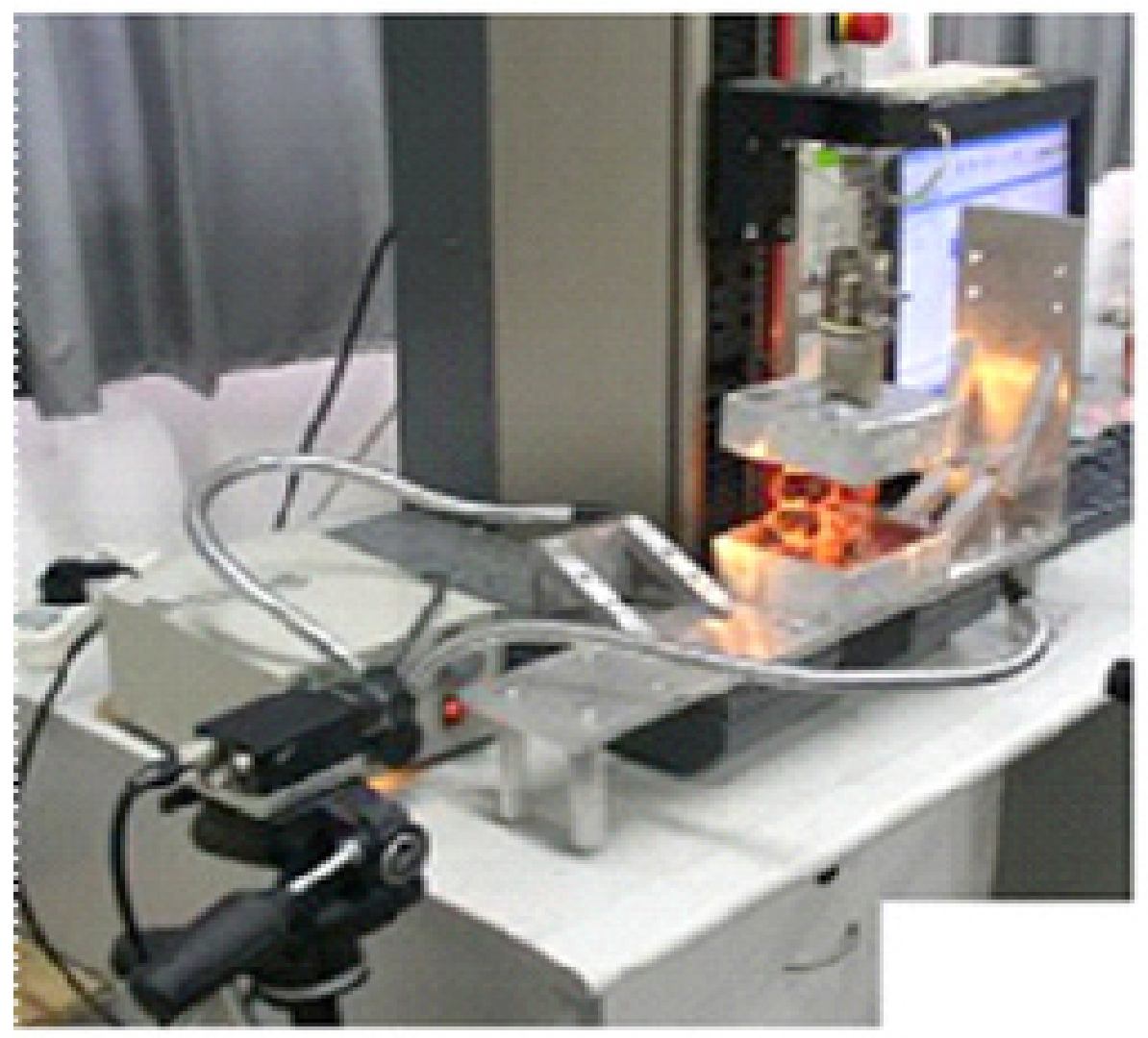

$126 \times 116 \mathrm{~mm}(72 \times 72 \mathrm{DPI})$ 
Domestic torsion testing machine (NT-100B) was used in the torsion experiment. Thoracic specimens were rotated left and right to $5^{\circ}$ to measure the torques on both sides using continuous digital strain gauge (YK14) (Fig. 1B).

\section{Fig. 1B}

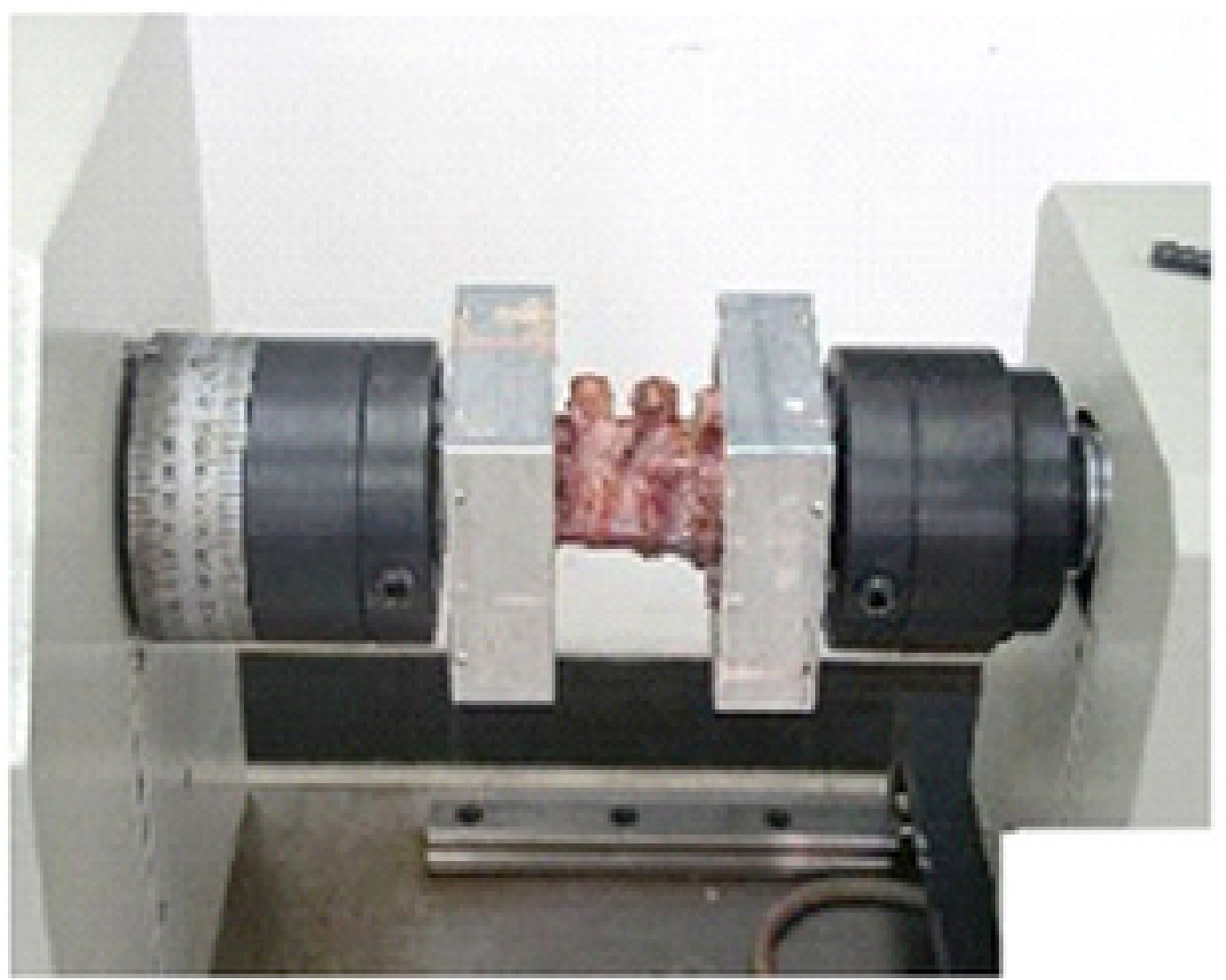

126x99mm (72 x $72 \mathrm{DPI})$

After that, every fixed centrum was cut and vertically pulled out on the universal testing machine at $5 \mathrm{~mm} /$ min (Fig. 1C). At all times, the specimens were kept 
moist with a saline spray.

\section{Fig. 1C}

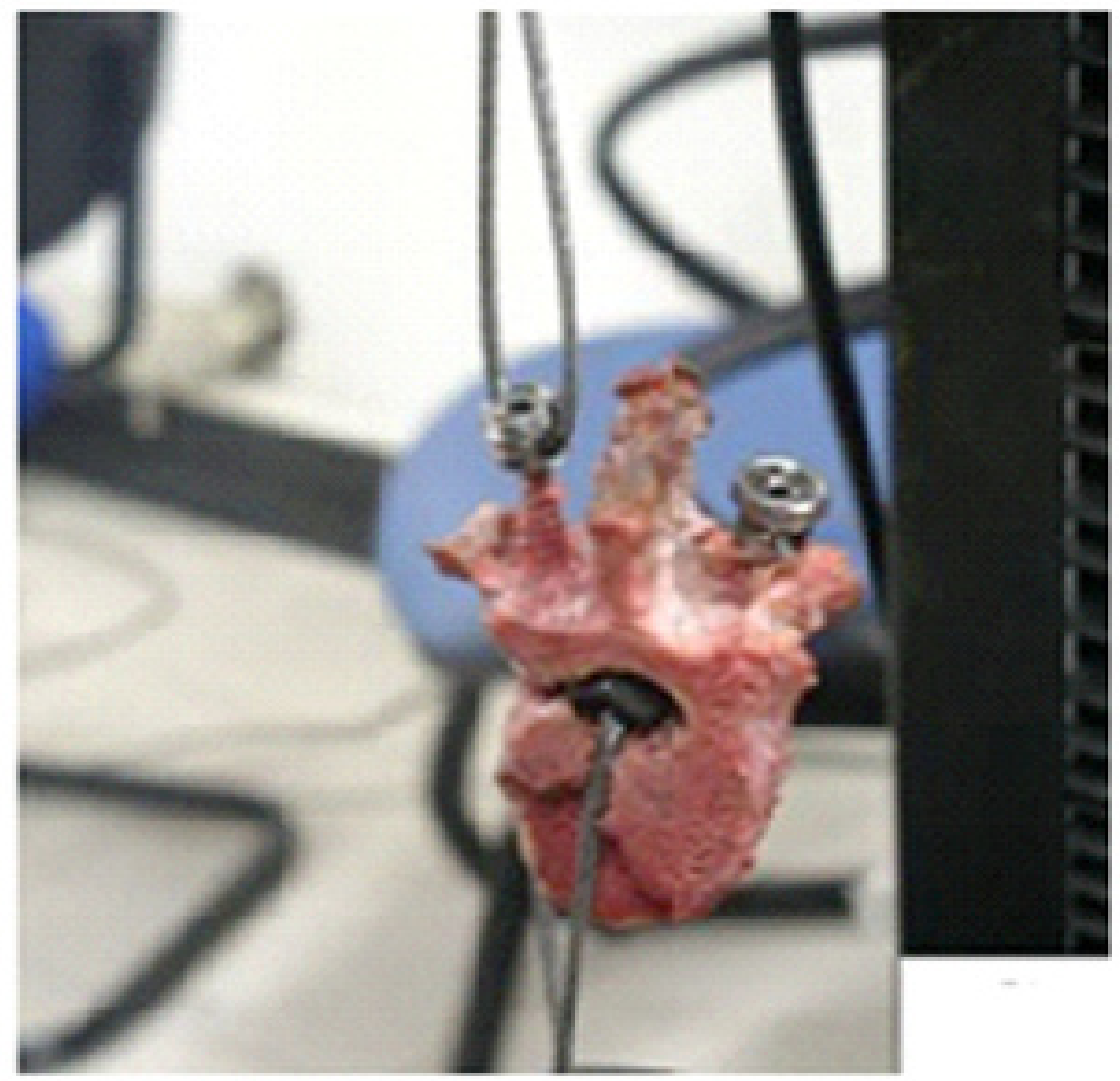

$127 \times 128 \mathrm{~mm}(72 \times 72 \mathrm{DPI})$

Method for thoracic spine fixation

After the biomechanical test for the 14 functional spinal units of intact thoracic spine, specimens were randomly divided into two groups to create damage models. Funnel technique and Magerl technique were used for fixation, respectively. In Funnel technique, the entry point was located superior to the intersection between the lateral border of the facet joint and the midpoint of the base of the transverse process. A $10 \mathrm{~mm}$ circle of cortical bone overlying the pedicle entry point was removed using rongeur. Then the cancellous bone was removed using curette from the back of pedicle continuously to the isthmus, which resulted in the formation of "funnel" configuration (Fig. 2). 
Fig. 2 The representative figure of Funnel technique.

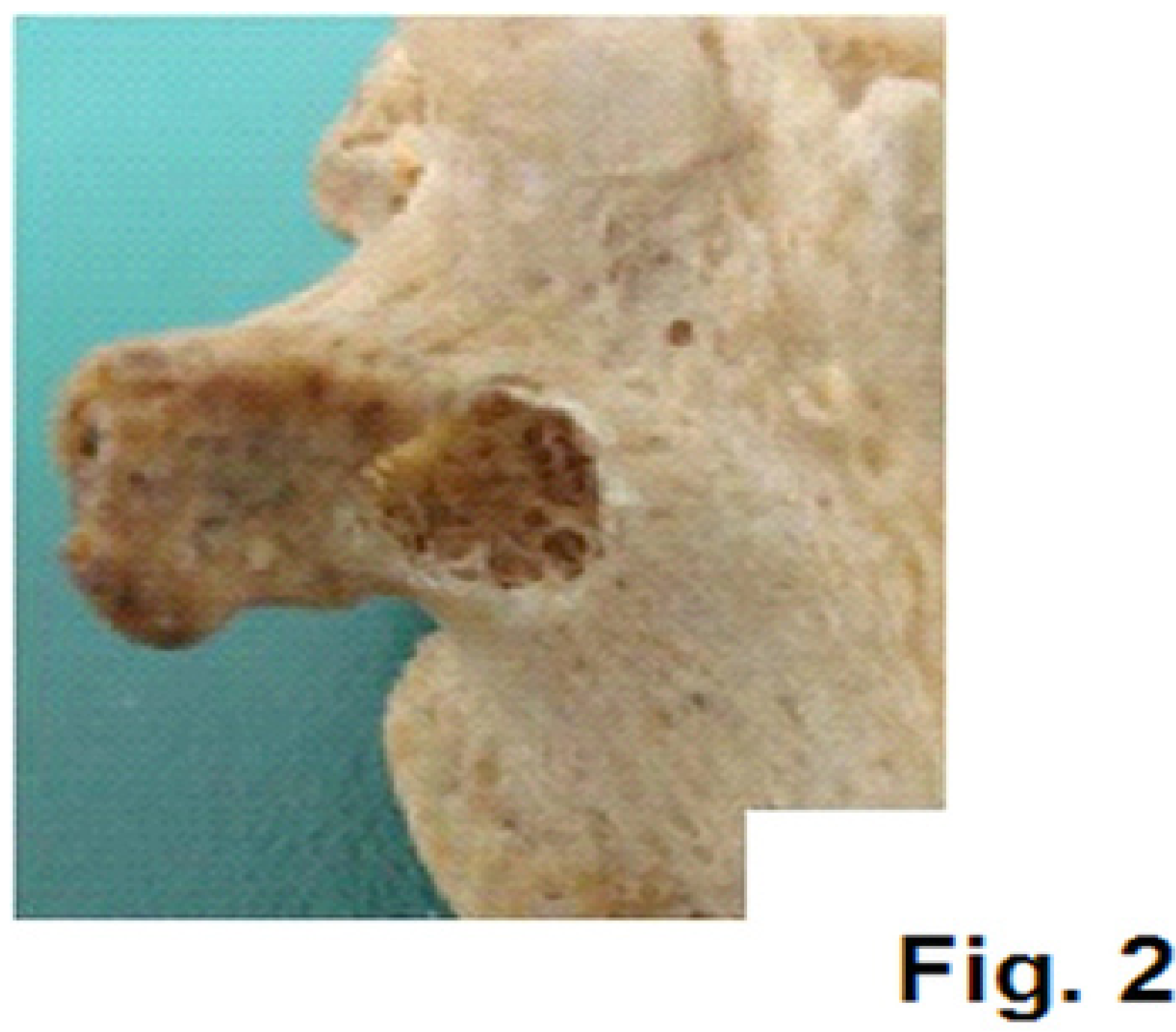

$126 \times 127 \mathrm{~mm}(72 \times 72 \mathrm{DPI})$

Tapping was conducted after testing the thoracic pedicle isthmus with a $2 \mathrm{~mm}$-diameter probe. And finally, screw was inserted and confirmed and then connected to rods. In Magerl technique, the intersection of the vertical line across the upper joint diapophysis and transverse bisector was chosen as the starting point. After removing a small amount of cortex, thoracic gag was directly punctured which followed by tapping and screwing. The screw was with a diameter of $4.5 \mathrm{~mm}$, length of $50 \mathrm{~mm}$. Screw tools were supplied by Depuy Co..

\section{Data analysis}

Data in this study were analyzed using SPSS software by calculating the mean value and the standard deviation. The difference was significant at the $\mathrm{P}<0.05$ level, and very significant at the $\mathrm{P}<0.001$ level. The group differences in stiffness between testing conditions were compared with q test. The relative values of displacement with $250 \mathrm{~N}$-loading exposure were compared under axial compression, flexion, extension and lateral flexion, where more displacements, the stiffness was small. While the relative values of torque were compared when the centrum making $1^{\circ}$ to $5^{\circ}$ rotation, where more torque, the stiffness was big. Curves were recorded during the pull out test until the screw was $10 \mathrm{~mm}$ out, and the maximum pullout forces were compared using onestudent unpaired t test.

\section{Results}

\section{Stiffness comparison under various conditions}

As shown in Table 1, under various conditions, stiffness of the fixing group was higher than the control group $(p<0.05)$, indicating the improvement of stiffness after fixation. 
Table 1 The comparison of displacement-stiffness under various state using different fixation techniques $(, \pm s)$

\begin{tabular}{|c|c|c|c|c|c|c|c|c|}
\hline Groups & $\mathrm{n}$ & $\begin{array}{l}A \quad x \quad i \quad a \quad 1 \\
\text { compression }\end{array}$ & $\begin{array}{l}\text { A n t e ri o r } \\
\text { flexion }\end{array}$ & $\begin{array}{l}\text { Posterior } \\
\text { extension }\end{array}$ & Left bending & $\begin{array}{l}\mathrm{R} \quad \mathrm{i} g \mathrm{~g} \\
\text { bending }\end{array}$ & $\begin{array}{l}\mathrm{L} e \mathrm{f} \quad \mathrm{t} \\
\text { rotation }\end{array}$ & $\begin{array}{l}\mathrm{R} \mathrm{ig} \mathrm{h} \mathrm{t} \\
\text { rotation }\end{array}$ \\
\hline Nomal & 14 & $69.31 \pm 5.07$ & $78.32 \pm 5.99$ & $49.41 \pm 6.93$ & $57.55 \pm 5.83$ & $58.83 \pm 5.81$ & $1.04 \pm 0.07$ & $1.07 \pm 0.65$ \\
\hline $\begin{array}{l}\text { F u n n e l } \\
\text { technique }\end{array}$ & 7 & $53.29 \pm 4.88$ & $42.05 \pm 4.51$ & $30.97 \pm 4.19$ & $49.63 \pm 4.73$ & $49.10 \pm 2.50$ & $1.76 \pm 0.12$ & $1.79 \pm 0.09$ \\
\hline M a g e r 1 & 7 & $54.25 \pm 3.99$ & $43.74 \pm 4.19$ & $31.40 \pm 3.25$ & $46.67 \pm 2.03$ & $50.27 \pm 2.96$ & $1.82 \pm 0.06$ & $1.72 \pm 0.36$ \\
\hline \multirow[t]{3}{*}{$\begin{array}{l}\text { technique } \\
\text { Total }\end{array}$} & 28 & $61.12 \pm 9.53$ & $60.61 \pm 18.75$ & $40.30 \pm 10.74$ & $52.85 \pm 6.81$ & $54.26 \pm 6.44$ & $1.42 \pm 0.39$ & $1.41 \pm 0.36$ \\
\hline & $\mathrm{F}$ & 40.956 & 158.51 & 36.791 & 13.505 & 13.946 & 300.746 & 341.309 \\
\hline & $\mathrm{P}$ & 0.786 & 0.554 & 0.887 & 0.270 & 0.640 & 0.180 & 0.097 \\
\hline
\end{tabular}

1. Axial compression, anterior flexion, posterior extension, left and right bending were described using relative displacement values $\left(10^{-3} \mathrm{~m}\right)$, left and right rotation were measured as relative torque with $5^{\circ}$ rotation $(\mathrm{N} . \mathrm{m})$.

2. F values were $q$ test results and $P$ values were the comparison of funnel and Magerl techniques.

However, there was no significant difference between the Funnel technique group and the Magerl technique group, indicating the little influence of Funnel technique towards the stiffness after removing part cortex and the spongy bone.
Comparison of the maximum axial pull-out force of the screw

Pull-out force curves were recorded for all 56 screws (Figure. 3a, 3b).

Fig. 3 Pullout strength curve for Magerl technique (A) and Funnel technique (B).

\section{Fig. $3 \mathrm{~A}$}

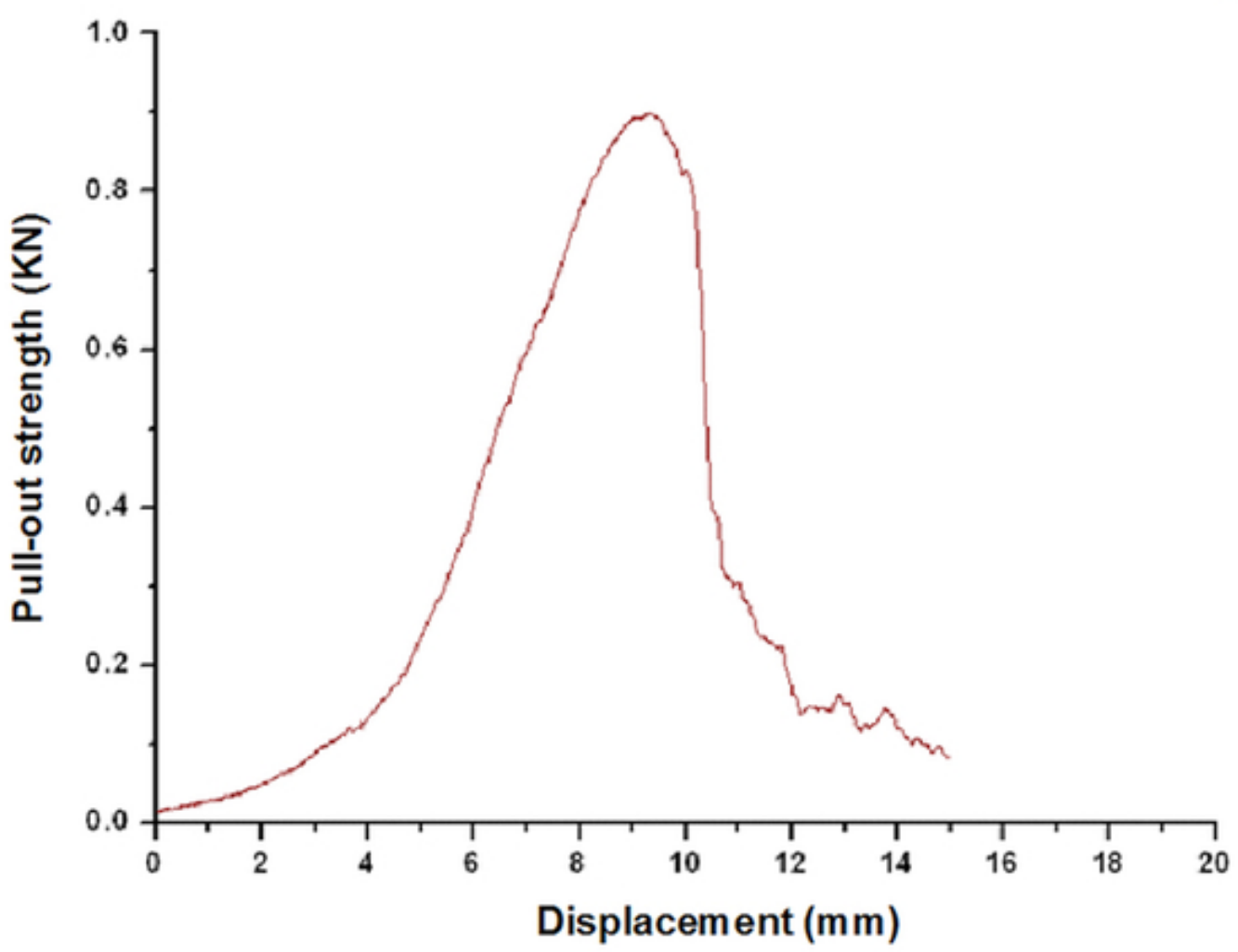

$210 \times 164 \mathrm{~mm}(72 \times 72 \mathrm{DPI})$ 
A typical curve is consisted of the rising and the declining period, and the peak value was the maximum pull-out force.

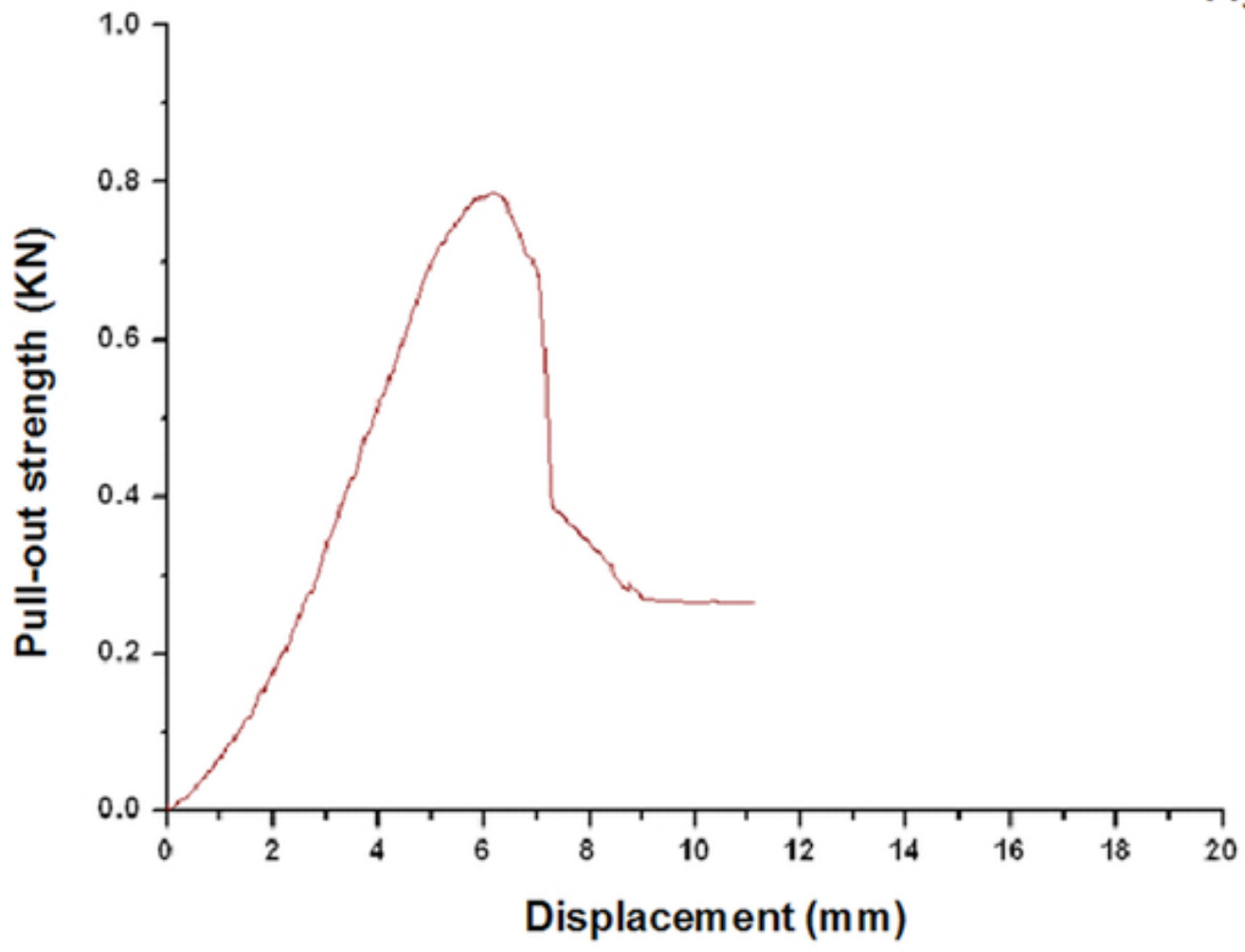

Fig. 3B

$$
210 \times 156 \mathrm{~mm}(72 \times 72 \mathrm{DPI})
$$

The average maximum pull-out forces in the two fixing groups were shown in Table 2. Independent sample t-test was used to analyze the two fixing groups, and the results showed that the maximum pull-out force in
Funnel technique group $(789.09 \pm 27.33)$ was lower than that in Magerl technique group (887.93 \pm 19.14$)$ with a $11.1 \%$ difference and the difference was statistically significant ( $\mathrm{t}=15.674, \mathrm{P}=0.000)$.

Table 2 The comparison of maximum pullout strength using different fixation techniques $(, \pm s, \mathrm{~N})$

\begin{tabular}{|l|l|l|l|l|}
\hline Groups & $\mathrm{n}$ & pull-out strength & $\mathrm{t}$ & $\mathrm{p}$ \\
\hline Funnel technique & 28 & $789.09 \pm 27.33$ & 15.674 & 0.000 \\
\hline Magerl technique & 28 & $887.93 \pm 19.14$ & & \\
\hline
\end{tabular}

\section{Discussion}

Thoracic pedicle has small circumference, complicated anatomic structure, and closely adjacent relationship with important blood vessel and viscera. Additionally, the anatomical landmarks for screw insertion were abnormal after fractura and degeneration. It was difficult for screw placement with the above characters which easily caused serious consequences after screw misplacement. Therefore, various screw placement techniques were applied to improve the accuracy and safety.

The traditional free-hand techniques (e.g. Magerl technique, Roy-Camile technique, etc.) for screw replacement highly depend on the experiences of the surgeons to judge anatomical landmarks, which was easy to operate on normal body. However, based on the anatomical abnormities (fractura, degeneration and scoliosis, etc.), it was difficulty to determine the starting 
point and correction angle, resulting in puncture of the pedicle. Screw insertion detected by direct visualization via a partial laminectomy exhibited higher accuracy than the traditional methods, but it took a long time and seriously affected the stability of the spinal column. Recently, thoracic pedicle side fixation technique (in-outin) has become a hot research topic for its high safety. Comparison research found that the pull-out strength with pedicle-rib screwing $(423.1 \pm 198.7 \mathrm{~N})$ was smaller than the pedicle screwing technique (783.3 199.5 $\mathrm{N})^{6}$. Computer-assisted technique was restricted for popularization by its high price and complexity. Therefore, development of a simple and safe screwing technique with good biomechanical property is very necessary but difficult in clinical work.

\section{Biomechanical study of the "Funnel technique"}

The present study compared the biomechanical properties and differences between Funnel technique and Magerl technique using fresh cadavers. In terms of fixing method, the following difference existed: For funnel technique, cortical bone was removed with rongeur to form the funnel configuration, where screw was inserted by direct visualization in the Funnel technique. For Magerl technique, drill was directly used for screw insertion. To ensure the quantity of samples, thoracic vertebrae was cut into FSU with same vertebrae body number and then randomly divided. Anterior column injury model was produced by simulating clinical practice, and the screw was correctly inserted by one surgeon who was experienced in cadaveric screw insertion and familiar with thoracic anatomy and fixation techniques. The length of screws used in this study was $50 \mathrm{~mm}$, and the insertion distances were $80 \%-90 \%$ of the distance between starting point and the vertebrae posterior marginal cortex, which was confirmed by X-ray ${ }^{7}$.

The stiffness of the two fixation groups was higher than the normal group, indicating the good stability of both techniques. Further study showed that thoracic vertebrae FSU stiffness in two fixation groups seemed to be no significantly different during anterior flexion, posterior extending, lateral bending and torsion under the normal physiological loading, . That indicated that removing of very tiny amount of cortex and cancellous bone has little impact on the stability of FSU, which is consistent with the previous report that about $80 \%$ of the caudocephalad stiffness depended on the pedicle rather than on the vertebral body ${ }^{2}$. The results in this study are also similar to the data by $\operatorname{Sun}^{8}$ with more FSU and improved bone removal technique.



Supplemental Figure: The brief description image of the construct.

Pull-out strength was also determined in this study. It was lower in Funnel technique group than Magerl group with significant difference, which indicates the pedicle screw axial pull-out strength decreased after partial removal of cortex and the spongy bone, which was different from previous studies in which the pullout strength was highly related to screw diameter, insertion depth and the bone density ${ }^{2} .60 \%$ of the pull-out strength of the pedicle screw depended on the pedicle rather than on the vertebral body, and it was useless or even harmful to increase the screw diameter and insertion depth for patient with osteoporosis. Same and different results were achieved by Weinatein ${ }^{9}$ and Daftari $^{10}$, respectively, which resulted from different measure methods, sample size and FSU selection.

In clinical works, funnel technique was used to estimate the entry structure of pedicle to identify the direction and location of screw placement. Results of clinical follow-up were satisfactory. No loose screws, no broken rod, and some patients with thoracic fracture had obtained bony union one year after the operation and come back for removal of fixation. However, 11.1\% difference of pullout strength still can not be ignored. So when using funnel technique, the cortex removal scale should be as small as possible to expose the pedicle isthmus for funnel formation. No blind probing, just inspect the depth of the opening and all four walls of 
the pedicle, in order to protect the cancellous bones to remain pullout strength. As to patients with normal bones, screw with larger diameter or increase the screw insertion depth is another choice. For patients with osteoporosis, bone mineral density and cortex were not as thick as normal, while absorbable cement such as polymethyl methacrylate or calcium phosphate can be used to improve the fixation strength. And the patients with great difficulty in screw placement, such as T3-T6 fracture and scoliosis, should be given the priority to use funnel technique.

\section{The shortcomings of the present study}

Our study has ignored the differences of pedicle diameter and length for $\mathrm{T} 7$ and $\mathrm{T} 9$ to insure the sample size due to the rare human specimen, which may cause impact on the pull-out strength study. So we have minimized the error by random grouping of all specimens. We expect that if we have enough fresh human specimens, better results will be gained aim to same position centrum.

\section{Summary}

Funnel technique partially removed the posterior bone for better probing and accurate screwing, and it efficiently avoided the complications related to improper screw placement. Biomechanical experiments indicated the same stiffness compared with the traditional technique without bone removal. However, the pullout strength was decreased with more bone removal. So we suggest the funnel technique be used in patients with great difficulty in screw placement as a complementary method of traditional technique.

\section{References:}

1. Yingsakmonkol W, Karaikovic E, Gaines RW. The accuracy of pedicle screw placement in the thoracic spine using the "Funnel Technique": part 1. A cadaveric study. Journal of spinal disorders \& techniques $2002 ; 15$ (6): 445-449.

2. Hirano T, Hasegawa K, Takahashi HE, et al. Structural characteristics of the pedicle and its role in screw stability. Spine 1997; 22 (21): 2504-2510.

3. Brantley AG, Mayfield JK, Koeneman JB, Clark KR. The effects of pedicle screw fit: an in vitro study. Spine 1994; 19 (15): 1752-1758.

4. Okuyama K, Abe E, Suzuki T, et al. Influence of bone mineral density on pedicle screw fixation: a study of pedicle screw fixation augmenting posterior lumbar interbody fusion in elderly patients. The Spine Journal 2001; 1 (6): 402-407.

5. Karaikovic EE, Yingsakmongkol W, Gaines Jr RW. Accuracy of cervical pedicle screw placement using the funnel technique. Spine 2001; 26 (22): 24562462.

6. Wei X, Hou SX, Shi YM, Wang XN, Zhang YP. Pullout strength of pedicle rib unit screws versus pedicle screws in the thoracic spine. Chinese journal of spine and spinal cord 2006; 16 (8): 623-625.

7. Krag MH, Beynnon BD, Pope MH, DeCoster TA. Depth of insertion of transpedicular vertebral screws into human vertebrae: effect upon screwvertebra interface strength. Journal of spinal disorders \& techniques 1988; 1 (4): 287-294.

8. Sun HS, Nen DH, Tang TS, et al. Biomechanical characteristics of modified hole preparation method for transpedical internal fixation. Chinese journal of clinical rehabilitation 2005; 9 (2): 60-62.

9. Weinstein JN, Rydevik BL, Rauschning W. Anatomic and technical considerations of pedicle screw fixation. Clinical Orthopaedics and related research 1992; 284 34-46.

10. Daftari TK, Horton WC, Hutton WC. Correlations between screw hole preparation, torque of insertion, and pullout strength for spinal screws. Journal of spinal disorders \& techniques 1994; 7 (2): 139-145. 